\title{
Research on Machining Algorithm of Ultra Precision Instrument Based on Computer Control
}

\author{
Juxia Xiong, ${ }^{1,, *}$, Jinzhao $\mathrm{Wu}^{2, b, *}$ \\ ${ }^{1}$ Chengdu Institute of Computer Application, Chinese Academy of Sciences, Chengdu, Sichuan, 610041, \\ China \\ ${ }^{2}$ University of Chinese Academy of Sciences, Beijing 100049, China \\ ${ }^{3}$ Guangxi Key Laboratory of Hybrid Computational and IC Design Analysis, Guangxi University for \\ Nationalities, Nanning, Guangxi, 53006, China \\ a email: xiongjuxia1107@163.com, ${ }^{\mathrm{b}}$ email: wjzgxun@163.com \\ *corresponding author
}

Keywords: Computer Control, Ultra Precision Machining, Algorithm Research

\begin{abstract}
Ultra precision machining is one of the main research contents of cutting-edge manufacturing technology. According to the theory of neutrino mechanics, the scale effect of materials at the scale of method is investigated. The ultra precision machining technology is studied by using molecular dynamics and finite element simulation technology, and the surface is measured and analyzed. Molecular dynamics is an important tool for nanofabrication. The molecular dynamics simulation model of diamond tool cutting for monocrystal silicon processing products is established, and the structure formed on the chip and surface of monocrystal silicon is established. The simulation is carried out when the formation depth and tool shape angle change. Material structure relationship and minimum cutting thickness are the theoretical basis of ultra precision machining. In this paper, the method of considering the scale effect is established, and the analytical formula of the minimum cutting thickness is derived. On this basis, the change of material stress, deformation and the minimum cutting thickness in ultra precision machining are studied. The results of this study show that the existing structural relationships well reflect the scale effect of material properties. The scale effect of material characteristic curve increases with the decrease of cutting depth, rake angle and cutting edge radius, while the friction coefficient decreases with the decrease of cutting depth, rake angle and cutting edge radius.
\end{abstract}

\section{Introduction}

The so-called ultra precision machining technology does not refer to specific machining methods, nor does it refer to higher-level machining technology than the given machining accuracy[1]. However, it is difficult to define ultra precision machining in terms of general terms of machining with high machining accuracy, what can be done in a specific era, and how to define ultra precision machining. Ultra precision is closely related to the level of processing and measuring technology in that era. In 1983, Professor kuguchi of Japan reviewed many cases of ultra precision machining, reviewed the current situation of ultra precision machining, and predicted the development trend. The past, present and future of ultra precision machining are systematically summarized. Reviewing the development of precision machining in the past decade, it is found that these curves reflect the development methods in this field. Ultra precision machining technology is the improvement of machining accuracy from. $1 \mathrm{~m}$ in the early 1960 s to. $1 \mathrm{~m}$ at present[2]. At present, it is developed at the atomic level. The latest electronic measurement, computer and other new machining technologies and new technology development bring new achievements.

\section{Fundamentals of Ultra Precision Machining Technology}

Ultra precision machining technology is an organized project with many influencing factors. 
Only through extensive research on various new technologies, comprehensive research and multifaceted improvement, can we break the accuracy limit that the existing processing technology cannot achieve. The conditions to realize ultra precision machining mainly include: ultra precision machining machine, ultra precision machining workpiece, ultra precision cutting tools, ultra precision machining environment, ultra precision machining instruments, ultra precision measurement and control technology [3]. Ultra precision machining technology is actually a comprehensive application of these technologies.

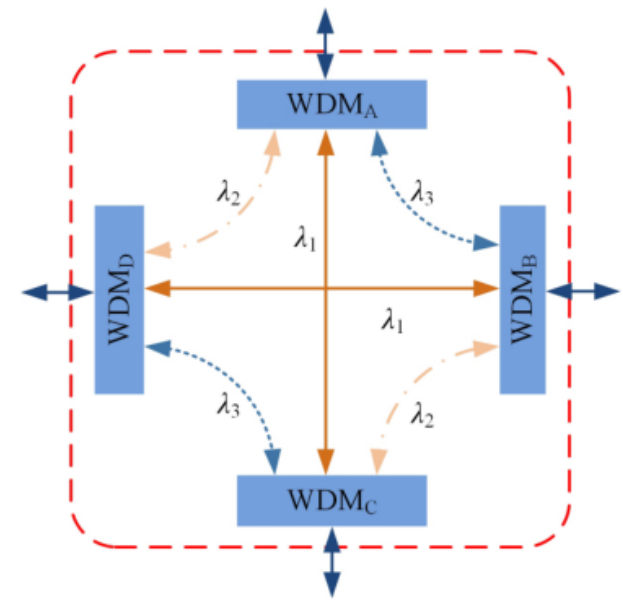

Figure 1 Four port all time all pass quantum router

\subsection{Ultra Precision Machining Machine}

Ultra precision machine tool is the main condition of ultra precision machining. In order to continuously improve the detection method, the machine tool has transferred from the traditional static rigid requirements to the dynamic and thermal rigid requirements[4]. In order to obtain various kinds of rigidity, there are many countermeasures, among which the choice of construction materials is of great concern. At present, the most commonly used material is natural stone, which is difficult to process. Later, it developed into artificial granite that can be built. In 1984, Japan developed all ceramic ultra precision lathes, and Professor Yuki kukawa successfully developed two ceramic lathes. Structural materials are still under development, glass ceramics, panel alloy, super panel are the leaders of all ultra precision machine tool structural materials. The development of ultra precision machine tools is an important prerequisite for the development of ultra precision machining. At present, the development trend of ultra precision machine tool technology is to combine the limitation, high precision, high efficiency, large-scale, miniaturization, functionality, processing and detection of machining. New principles, new methods and new materials have been developed in the direction of structural versatility and modularity. Generally speaking, it is an extreme manufacturing technology direction.

\subsection{Ultra Precision Machining Tools and Workpiece Materials}

Ultra precision machining tool is one of the key technologies of ultra precision machining. The chip can be removed accurately under the condition of submicron and nanometer. Therefore, the cutting edge of the cutting tool should be very sharp[5]. Diamond cutting machine and diamond wheel are widely used in ultra precision machining and become the symbol of ultra precision machining. That technology is maturing. The United States and Japan are in a leading position in this field. The research of AVIC 628, Harbin Institute of technology and other institutions is in progress. Ultra precision turning speed is high, conveying capacity is small, back eat a small number of knives. Therefore, the requirements for tool materials are high hardness, wear resistance, high elasticity, long life and durability. In order to obtain a good processing surface, the processed materials need good adhesion resistance, small chemical affinity and low friction coefficient. At present, the tool materials used in ultra precision turning at home and abroad include ceramic tools, polycrystalline diamond turning tools, single crystal diamond turning tools, polycrystalline cubic 
boron nitride turning tools. Diamond tools are used for ultra precision cutting. The results of abrasion experiments carried out by the American Research Institute show that diamond tools cut off the non-metal[6]. The roughness of the cutting section is still below $01 \mathrm{~m}$ after the cutting distance is $20 \mathrm{~km}$. Therefore, the products processed with diamond tools have very good consistency.

Table 1 Table of computer encryption system

\begin{tabular}{|c|c|}
\hline $\begin{array}{l}\text { Restriction Conditions of Industrial } \\
\text { Integration Theorem }\end{array}$ & Corresponding selected financial indicators \\
\hline sales revenue & $\begin{array}{c}\text { Main Business Income-Return of Sales-Sales Loan Discount and } \\
\text { Discount }\end{array}$ \\
\hline Variable cost & $\begin{array}{c}\text { Operating Cost+Business Tax and Additional+Sales } \\
\text { Cost+Management Cost+Financial Cost }\end{array}$ \\
\hline
\end{tabular}

\section{Research on the Theory of Ultra Precision Machining}

The precision requirement of ultra precision machining is higher and higher, and the precision margin of machine tool is very small. In this case, it is difficult to improve only by improving the original technology, so we should start from the processing principle to find solutions. Ultra precision machining not only has the general characteristics of traditional machining, such as large deformation, high deformation rate and important temperature change, but also has the special characteristics of machining, which is mainly manifested in the proportion effect and the minimum cutting thickness[7]. At present, the research on the theory of method scale processing is in the exploratory stage.

\subsection{Mesoscale Size Effect}

In traditional cutting, the cutting force and cutting energy will decrease with the decrease of the amount of material removed, but the experiment shows that the cutting energy ratio will increase abnormally when the cutting feed is reduced to micron level. The scale effect has been verified by many experiments, but it has not been fully explained. Therefore, the mechanical properties of materials are the basis of studying the mechanism of scale processing[8]. According to the theory of mechanical strain gradient and its experimental study, the mechanical properties of materials show a strong scale effect. Based on the positioning mechanism, this scale effect is revealed and good results are obtained. Since the deformation scale of the material treated by the method scale is correct to be micron, it can be inferred that the size effect of the method scale is caused by the size effect of the mechanical properties of the material under the method scale. The effect of deformation scale is analyzed by finite element simulation.

\subsection{Minimum Cutting Thickness}

The tip radius is a very important feature of methyl cutting which is different from the previous cutting when the tool passes through. When the cutting depth increases gradually, when the cutting radius is far less than the cutting edge radius, the cutting depth only has elastic deformation when the tool passes through. When the cutting depth is close to the cutting edge radius, the methyl cutting process is divided into three stages. The actual cutting front angle is negative, resulting in back scraping. Plastic deformation; cutting occurs only when the cutting depth increases further[9]. The phenomenon of minimum cutting thickness reflects the mechanical properties of materials and the influence of tool tip radius. Precision calculation is the key to study the method level large-scale machining mechanism. The cutting radius is an important factor affecting the minimum cutting thickness. The minimum cutting thickness can be accurately calculated by finite element simulation, and the influence of tool tip radius, material mechanical properties and other factors can be analyzed. 


\section{Molecular Dynamics Simulation}

The tip radius is a very important feature of methyl cutting which is different from the previous cutting when the tool passes through. When the cutting depth increases gradually, when the cutting radius is far less than the cutting edge radius, the cutting depth only has elastic deformation when the tool passes through. When the cutting depth is close to the cutting edge radius, the methyl cutting process is divided into three stages. The actual cutting front angle is negative, resulting in back scraping. Plastic deformation; cutting occurs only when the cutting depth increases further. The phenomenon of minimum cutting thickness reflects the mechanical properties of materials and the influence of tool tip radius. Precision calculation is the key to study the method level large-scale machining mechanism[10]. The cutting radius is an important factor affecting the minimum cutting thickness. The minimum cutting thickness can be accurately calculated by finite element simulation, and the influence of tool tip radius, material mechanical properties and other factors can be analyzed.

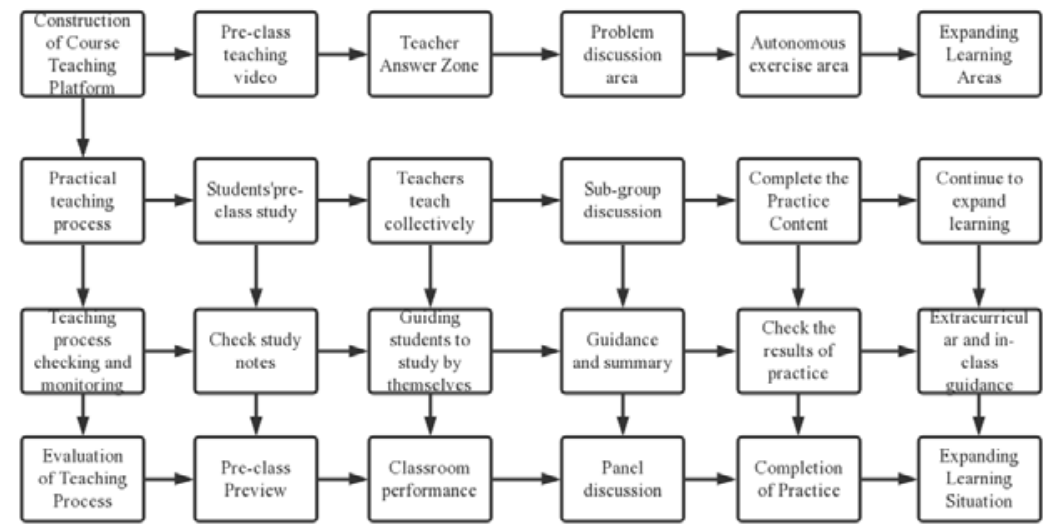

Figure 2 Precision machining algorithm mode

\section{Conclusion}

Work, as a sharing and combining monocrystal silicon material, diamond as tool, Tersoff scalar potential as scalar potential between atoms in the processed object, and Morse, as scalar potential between tool and work object, use scalar potential to establish a molecular simulation cutting simulation system. By observing the simulation results, the effect of nano machining technology is explained. According to the simulation results, monocrystal silicon of hard and brittle materials can be removed plastically in nano cutting. From the lattice point of view, the conclusion is that the tool pre silicon in the machining process is subject to some traceless lattice, local elastic deformation, displacement, plastic deformation and amorphous formation. After the tool passes, the workpiece surface is elastically recovered and lattice reconstructed to form a machined surface with multiple roll positions. In addition, the simulation tests of different cutting conditions are carried out. As a result, due to the reduction of the cutting depth, the generated surface rotation depth does not decrease. It is assumed that the thickness of the deformation layer of the pre machined surface has a minimum limit. At the same time, clear tools, better chip formation, similar to the traditional macro cut. When the tool has a large negative rake angle, the volume of workpiece changes greatly before and after machining. According to the analysis, the working phase changes in machining. By extracting the simulated temperature, the temperature distribution in the workpiece can be observed clearly. The temperature of the amorphous in the cut-off region is the highest, the atoms forming plastic deformation and translocation continue, and the atoms far away from the cut-off region have the lowest temperature.

\section{Acknowledgements}

1) This research has been financed by the National Natural Science Foundation of China " Error 
analysis and control of semi-algebraic model detection method" (61772006);

2) The Science and Technology Major Project of Guangxi "Research and Application Demonstration of Key Technologies for Intelligent Ship Networking in Beibu Gulf” (AA17204096);

3) The Key Research and Development Project of Guangxi "DPA-proof full asynchronous RSA security crypto chip: design methods, tools and prototypes” (AB17129012);

4) The Special Fund for Bagui Scholars of Guangxi "Control system design and verification” (2017);

5) The Promotion Project of Basic Faculties for Young and Middle-aged College Teachers in Guangxi "Research on Formal Analysis Method of Hybrid System Based on Polyhedron Projection and Segmentation” (2017KY0174);

6) The Promotion Project of Basic Faculties for Young and Middle-aged College Teachers in Guangxi “Common Sense Dynamic Logic Reasoning and Its Application” (2018KY0164).

\section{References}

[1] Dai H, Chen G, Cong Z, et al. A numerical study of ultraprecision machining of monocrystalline silicon with laser nano-structured diamond tools by atomistic simulation, vol. 393, pp. 405-416, 2017.

[2] Qinglong An, Jiaqiang Dang, Gongyu Liu, A new method for deburring of servo valve core edge based on ultraprecision cutting with the designed monocrystalline diamond tool. Science China Technological Sciences, 2019.

[3] Xun Chen, Akinjide Olufemi Oluwajobi. Characterization of Atomic Surface Roughness in Nanometric Machining Molecular Dynamics (MD) Simulations. Current Nanoscience, vol. 13, no. 1, 2017.

[4] Faezeh Kazemi, Georg Boehm, Thomas Arnold. Development of a model for ultra recise surface machining of $\mathrm{N} \| \mathrm{K} 7 \AA$ using microwave " riven reactive plasma jet machining. Plasma Processes and Polymers, 2019.

[5] Viktor Molnar. Indirect Impacts of Drastic Scrap Rate Reduction on Costs of Production Process in Precision Machining. Solid State Phenomena, vol. 261, pp. 487-494, 2017.

[6] Jong-Su Kim, Hongseok Youn, Bongchul Kang. Ultra-precision 3 DOF tilting stage for workpiece setup of scalable micro-pattern machining. International Journal of Precision Engineering \& Manufacturing, vol. 18, no. 8, pp. 1103-1109, 2017.

[7] Duo Li, Bo Wang, Zhen Tong, On-machine surface measurement and applications for ultraprecision machining: a state-of-the-art review. International Journal of Advanced Manufacturing Technology, vol. 104, no. 1-4, 2019.

[8] M. Azizur Rahman, Mustafizur Rahman, A. Senthil Kumar. Influence of relative tool sharpness (RTS) on different ultra-precision machining regimes of $\mathrm{Mg}$ alloy. International Journal of Advanced Manufacturing Technology, vol. 96, no. 11, pp. 1-19, 2018.

[9] Yonggou Lou, Hongbing Wu. Improving machinability of titanium alloy by electro-pulsing treatment in ultra-precision machining. International Journal of Advanced Manufacturing Technology, vol. 93, no. 2015, pp. 2299-2304, 2017.

[10] Bo Xue, Yanquan Geng, Yongda Yan, Effects of AFM tip wear on evaluating the surface quality machined by ultra-precision machining process. International Journal of Advanced Manufacturing Technology, pp. 1-13, 2019. 